\title{
Editorial
}

\section{Numerical Linear Algebra in Signal Processing Applications}

\author{
Nicola Mastronardi, ${ }^{1}$ Gene H. Golub, ${ }^{2}$ Shivkumar Chandrasekaran, ${ }^{3}$ Marc Moonen, ${ }^{4}$ \\ Paul Van Dooren, ${ }^{5}$ and Sabine Van Huffel ${ }^{4}$ \\ ${ }^{1}$ Istituto per le Applicazioni del Calcolo "M. Picone", sede di Bari, Consiglio Nazionale delle Ricerche Via G. Amendola 122/D, \\ I-70126 Bari, Italy \\ ${ }^{2}$ Department of Computer Science, Stanford University, Gates Building 2B, Room 280, Stanford, CA 94305-9025, USA \\ ${ }^{3}$ Department of Electrical and Computer Engineering, University of California, Santa Barbara, CA 93106, USA \\ ${ }^{4}$ Department of Electrical Engineering, Katholieke Universiteit Leuven, Kasteelpark Arenberg 10, bus 2446, \\ B-3001 Leuven-Heverlee, Belgium \\ ${ }^{5}$ Department of Mathematical Engineering, Catholic University of Louvain, Bâtiment Euler (A.202), \\ Avenue Georges Lemaitre 4, B-1348 Leuven-Heverlee, Belgium
}

Received 27 September 2007; Accepted 27 September 2007

Copyright @ 2007 Nicola Mastronardi et al. This is an open access article distributed under the Creative Commons Attribution License, which permits unrestricted use, distribution, and reproduction in any medium, provided the original work is properly cited.

The cross-fertilization between numerical linear algebra and digital signal processing has been very fruitful in the last decades. In particular, signal processing has been making increasingly sophisticated use of linear algebra on both theoretical and algorithmic fronts. The interaction between them has been growing, leading to many new algorithms. In particular, numerical linear algebra tools, such as eigenvalue and singular value decomposition and their higher-extensions, least squares, total least squares, recursive least squares, regularization, orthogonality and projections, are the kernels of powerful and numerically robust algorithms in many signal processing applications.

This special issue contains contributions written by experts of signal processing, computer engineering, and numerical analysis, providing an account of the main results in this interdisciplinary field. Most of the papers are devoted to applications of numerical linear algebra algorithms for solving signal processing problems. Nevertheless, few of them are more theoretically oriented, and describe algorithms for solving linear algebra problems involving structured matrices and tensors, frequently encountered in a variety of signal processing applications.

In the paper by H. Reza Bahrami et al., the effect of eigenvalues distribution of spatial correlation matrices on the capacity of frequency-flat and frequency-selective channels is first investigated. Then, a practical scheme, known as linear precoding, is introduced. It can enhance the ergodic capacity of the channel by changing the eigenstructure of the channel, applying a linear transformation. The structures of precoders using eigenvalue decomposition and linear algebra techniques are derived and their similarities from an algebraic point of view are shown.

Numerical methods for finding the maximal symmetric positive definite solution of the nonlinear matrix equation $X=Q+L X^{-1} L^{T}$, where $Q$ is symmetric positive definite and $L$ is nonsingular, are studied in the article by $\mathrm{P}$. Benner and $\mathrm{H}$. Faßbender. Such equations arise, for instance, in the analysis of stationary Gaussian reciprocal processes over a finite interval. Its unique largest positive definite solution coincides with the unique positive definite solution of a related discrete-time algebraic Riccati equation.

I. Drori, in his paper, presents a method which takes advantage of the sparsity of the wavelet representation of the nuclear magnetic resonance (NMR) spectra and reconstructs the spectra from partial random measurements of its free induction decay. This is done by solving an optimization problem. In the settings of interest, the underlying solution is sparse with a few nonzero entries. For large practical systems, a good approximation of the solution can be obtained by iterative thresholding algorithms, running much more rapidly than general solvers. The applicability of this approach to fast multidimensional NMR spectroscopy is shown.

The paper by P. Favati et al. deals with image restoration problems. Among the many regularization methods used for handling the problem, iterative methods have been shown to be effective. The authors propose inverse preconditioners with a band Toeplitz structure for solving linear systems having band block Toeplitz structure with band Toeplitz blocks, 
in the case of a blurring function defined by space invariant and band-limited point spread function.

A survey on the definitions and use of rank-revealing matrix decompositions in single-channel noise reduction algorithms for speech signals is given in the article by P. C. Hansen and S. H. Jensen. The proposed algorithms are based on the rank-reduction paradigm and, in particular, signal subspace techniques. The focus is on practical working algorithms, using both diagonal (eigenvalue and singular value) decompositions and rank-revealing triangular decompositions. In addition, it is shown how the subspace-based algorithms can be analyzed and compared by means of simple FIR filter interpretations.

In the paper by H. Ji and C. Fermüller, an approach which utilizes color information in estimating optical flow is presented. Although color images do not provide more geometric information than monochromatic images in the estimation of optic flow, they contain additional statistical information. By utilizing the technique of instrumental variables, the authors show how to robustly correct bias from multiple noise sources without computing the parameters of the noise distribution.

In the paper by F. Kaltenberger et al., a low-complexity algorithm for the implementation of a geometry-based channel model on a hardware channel simulator is presented. The proposed algorithm takes advantage of the limited numerical precision of the channel simulator by using a truncated subspace representation of the channel transfer function based on multidimensional discrete prolate spheroidal (DPS) sequences. The DPS subspace representation offers two advantages. Firstly, only a small subspace dimension is required to achieve the numerical accuracy of the hardware channel simulator. Secondly, the computational complexity of the subspace representation is independent of the number of multipath components.

The paper by V. Kekatos et al. deals with adaptive equalization of wireless systems operating over time-varying and frequency-selective multiple-input multiple-output channels. A novel equalization structure is proposed. The equalizer filters, as well as the ordering by which the streams are extracted, are updated based on the minimization of a set of least squares cost functions in a BLAST-like fashion. To ensure numerically robust performance of the proposed algorithm, Cholesky factorization of the equalizer input autocorrelation matrix is applied.

In the article by M. Ladisa et al., a reliable and automatic method is applied to crystallographic data for tissue typing. The technique is based on canonical correlation analysis, a statistical method which makes use of the spectral-spatial information characterizing X-ray diffraction data measured from bone samples with implanted tissues.

The paper by J. Liang et al., proposes a new cumulantbased algorithm to jointly estimate four-dimensional source parameters of multiple near-field narrowband sources. Firstly, this approach proposes a new cross-array, and constructs five high-dimensional Toeplitz matrices using the fourth-order cumulants of some properly chosen sensor outputs; secondly, it forms a parallel factor model in the cumulant domain using these matrices, and analyzes the unique low-rank decomposition of this model; thirdly, it jointly estimates the frequency, two-dimensional directions-of-arrival, and range of each near-field source from the matrices via the low-rank three-way array decomposition.

In the article by Z. Nikolić et al., the transformation of selected linear algebra algorithms from floating point to fixed point arithmetic is analyzed. Moreover, real-time requirements and performance between the fixed point digital signal processors (DSPs) and floating point DSP algorithm implementations are compared. Its also introduced an advanced code optimization and an implementation by DSP-specific, fixed point $\mathrm{C}$ code generation.

The paper by $\mathrm{H}$. Semira et al. proposes a new algorithm for the direction-of-arrival (DOA) estimation of $P$ radiating sources. Unlike the classical subspace-based methods, the proposed algorithm involves the building of the signal subspace from the residual vectors of the conjugate gradient (CG) method. This approach is based on the same recently developed procedure which uses a noneigenvector basis derived from the auxiliary vectors (AVs). The AV basis calculation algorithm is replaced by the residual vectors of the CG algorithm. Then, successive orthogonal gradient vectors are derived to form a basis of the signal subspace.

In the paper by M. T. Signes Pont et al., a method to improve the calculation of functions which demand a great amount of computing resources is presented. The method is based on the choice of a weighted primitive which enables the calculation of function values under the scope of a recursive operation. When tackling the design level, the method turns out to be suitable for developing a processor which achieves a satisfying tradeoff between time delay, area costs, and stability. The method is particularly suitable for the mathematical transforms used in signal processing applications.

In the article by J. Yang et al., a robust adaptive algorithm for generalized eigendecomposition problems that arise in modern signal processing applications is proposed. To that extent, the generalized eigendecomposition problem is reinterpreted as an unconstrained nonlinear optimization problem. Starting from the proposed cost function and making use of an approximation of the Hessian matrix, a robust modified Newton algorithm is derived. A rigorous analysis of its convergence properties is presented by using stochastic approximation theory.

The paper by $\mathrm{X}$. Zhang and $\mathrm{D}$. Xu, links the polarizationsensitive-array signal detection problem to the parallel factor (PARAFAC) model, an analysis tool rooted in psychometrics and chemometrics. Exploiting this link, it derives a deterministic PARAFAC signal detection algorithm. The proposed PARAFAC signal detection algorithm fully utilizes the polarization, spatial and temporal diversities, and supports small sample sizes. The PARAFAC algorithm does not require direction-of-arrival information and polarization information, so it has blind and robust characteristics.

\section{ACKNOWLEDGMENTS}

The guest editors would like to thank all the authors who submitted papers to this special issue and many colleagues who took part in the review process. The efforts of the 
reviewers and their constructive criticism and remarks have led to considerable improvement of the papers and the overall quality of the issue. We also appreciate the efforts of both the authors of the included papers and the reviewers to comply with the submission and revision timeline. Finally, we would like to thank the Editorial Office of EURASIP JASP and the Professors M. Moonen and A. H. Sayed (the former and current Editor-in-Chief, resp.) for their continuous and valuable support.

\author{
Nicola Mastronardi \\ Gene H. Golub \\ Shivkumar Chandrasekaran \\ Marc Moonen \\ Paul Van Dooren \\ Sabine Van Huffel
}

